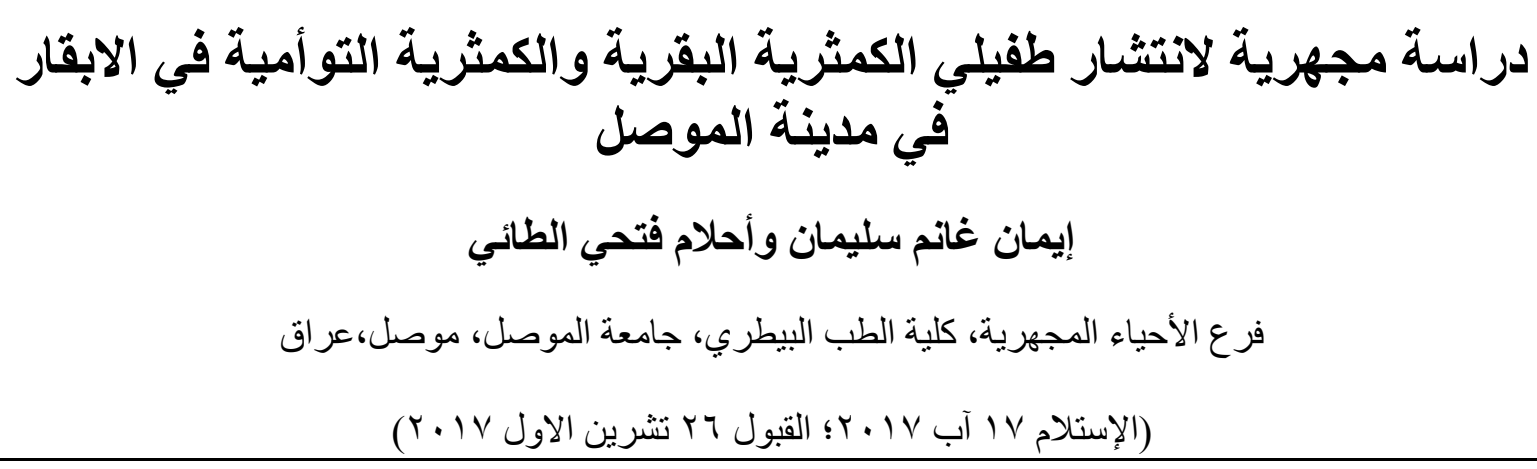

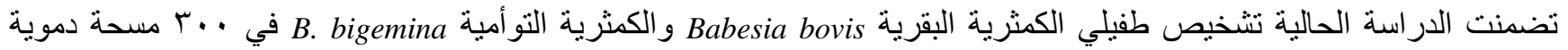

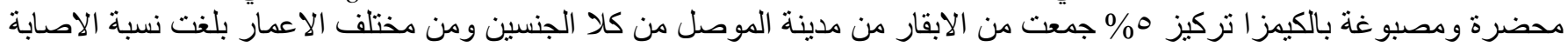

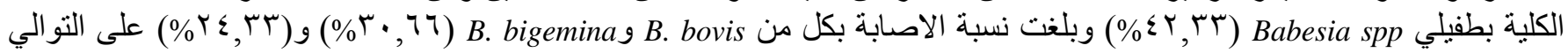

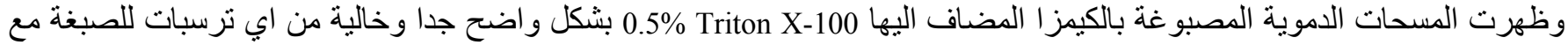

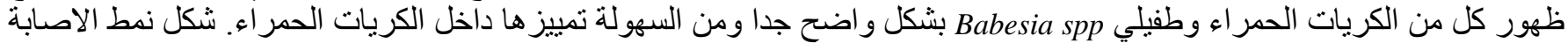

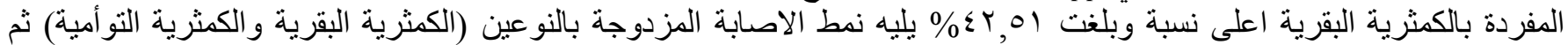

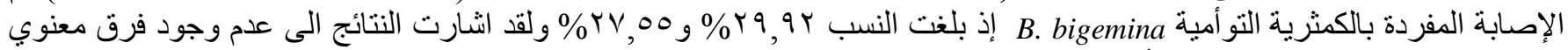
في نسبة الإصـابة بين إناث وذكور الأبقار وبين الفئات العمرية المختلفة وذللك عند مستوى معنوية

\title{
Microscopic study for prevalence of Babesia bovis and Babesia bigemina in cattle in Mosul city
}

\section{E.G. Suleiman and A.F. Altaee}

Department of Microbiology, College of Veterinary medicine, University of Mosul, Mosul, Iraq

\section{Abstract}

The current study included examination of Babesia bovis and Babesia bigemina in 300 blood smears stained with 5\% of Giemsa stain which collected from cattle in Mosul city from both sexes with different ages, the total percentage of infection with Babesia spp was $42.33 \%$ and the percentage of B. bovis and B. bigemina $30.66 \%$ and $24.33 \%$. The blood smears which stained with Giemsa stain contain 0.5\% Triton X-100 appeared cleaner and easily recognized the blood cells and parasites. High percentage of infection was appeared with single infection with $B$. bovis was $42.51 \%$ followed by mixed infection with B. bovis and B. bigemina and infection with B. bigemina only was $29.92 \%$ and $27.55 \%$. The results of this study showed no significant differences in the percentage of infection between males and females of cattle and different groups of ages at the significant level $\mathrm{p}<0.05$.

Available online at http://www.vetmedmosul.org/ijvs

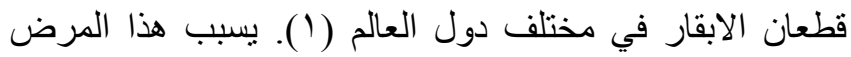
المقدمة

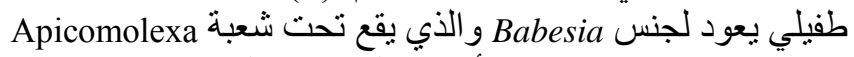

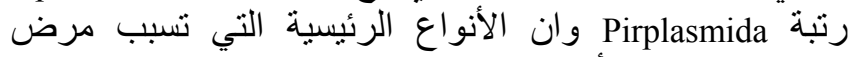

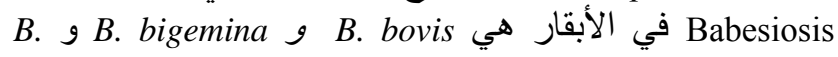

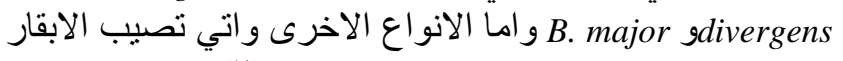

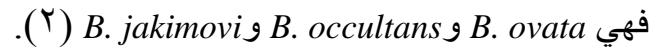

يعد مرض Babesiosis او بما بسمى بداء الكمثريات او حمى تكساس Piroplasmosis

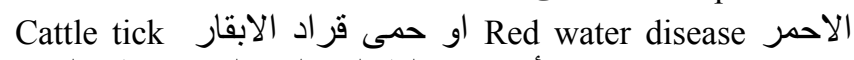
fever

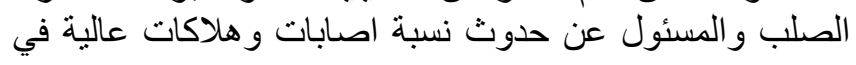


التعليمي /كلية الطب البطري /جامعة الموصل و كلية الزراعة

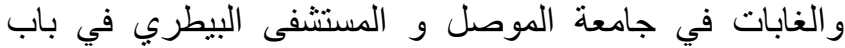

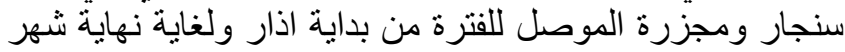

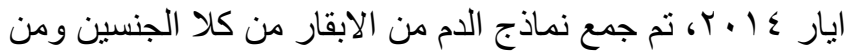

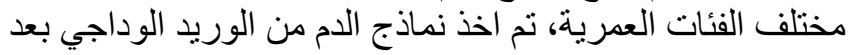

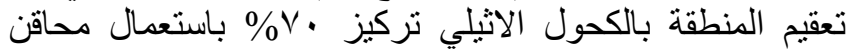

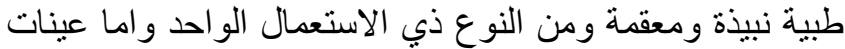

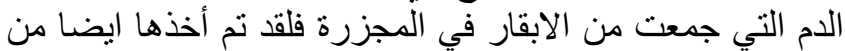

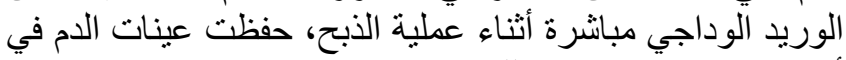

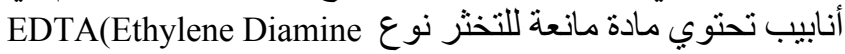
Tetera acetic Acid)

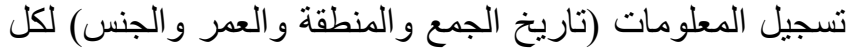

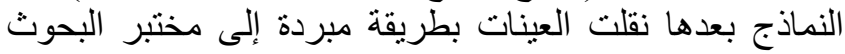

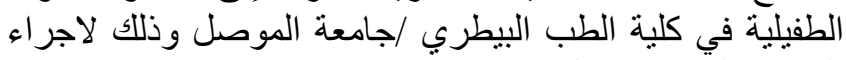

الفحص المختبري عليها.

تحضير مسحات دموية رقيقة Thin blood smears وذللك باخذ

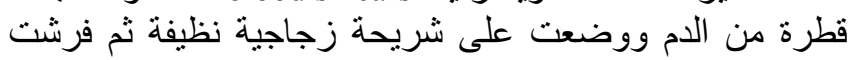

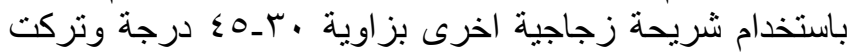

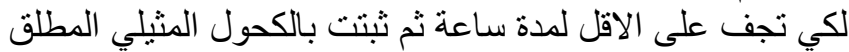

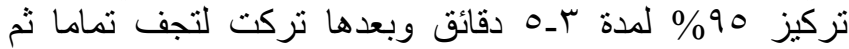

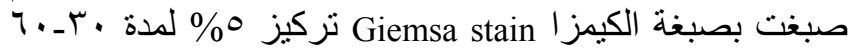

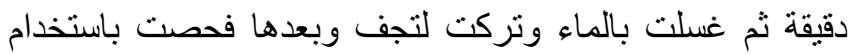
المجهر الضوئي نوع Olumpus بواسطة العدسة الزيتية وبقوة بأنية تكبير X100 (1) (1).

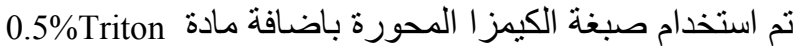

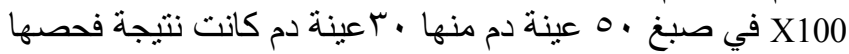

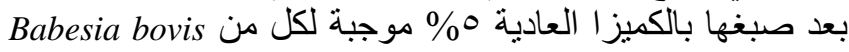

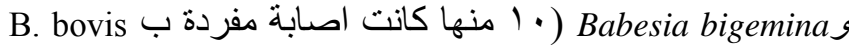

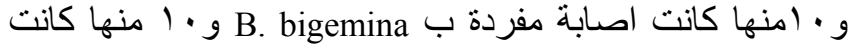

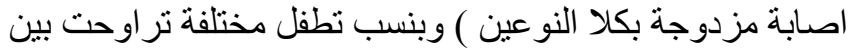

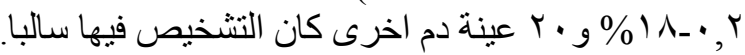

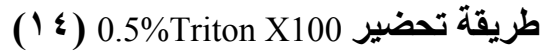

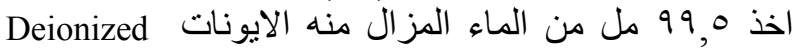

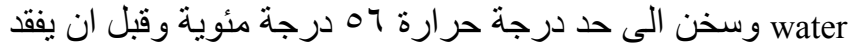

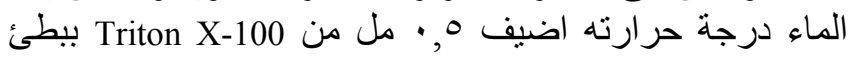

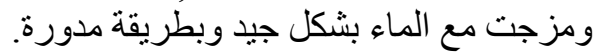

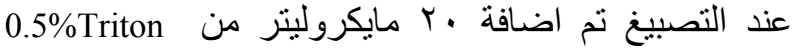

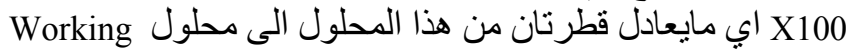
.Giemsa Stain

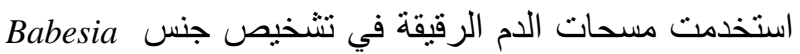

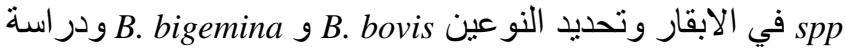
المواصفات الثكلية وتحديد الموقع داخل الكريات الحمر اء وقياس ودياس
يعد كل من B. bigemina و من اكثر الانواع B. B. Bovis

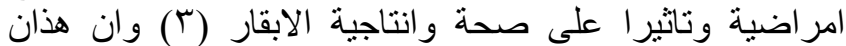

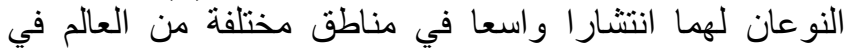

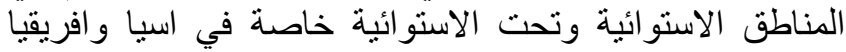

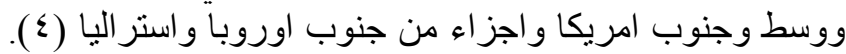

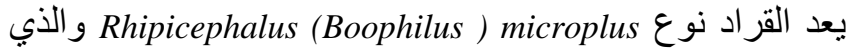

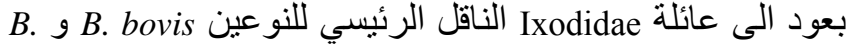

(०) bigemina

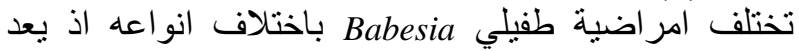

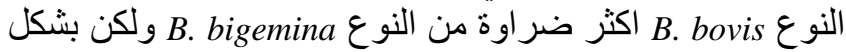

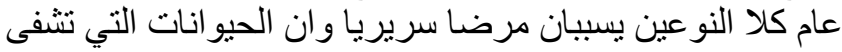

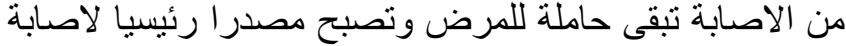

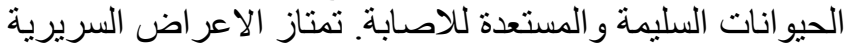

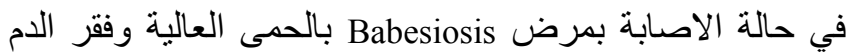
والبيلة الهيموكلوبينية والترنح وقد يحدث الإجهاض في الإني الابقار

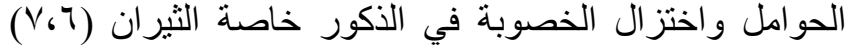

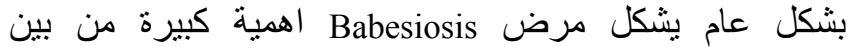

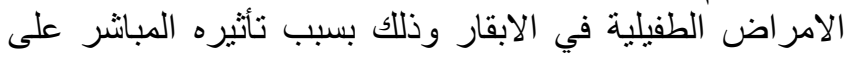

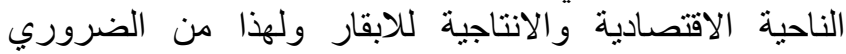

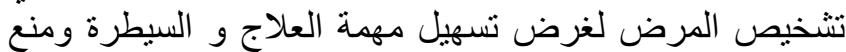

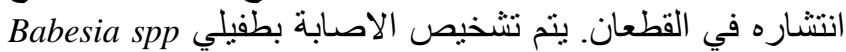
وذللك من خلال ملاحظة العلامات السريرية اضافة الى تلى تاريخ

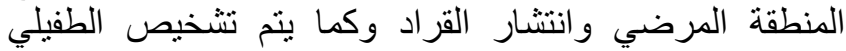

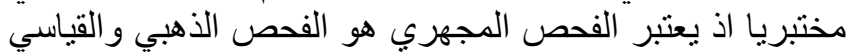

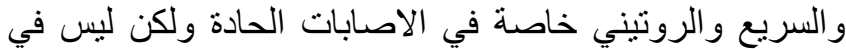

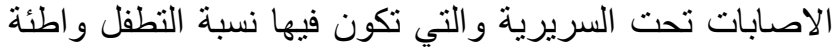

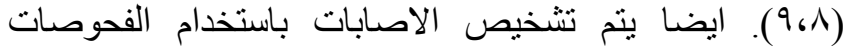
المصلية وهي فحوصات غير مباشرة تستخدم لتحديد الاجسام

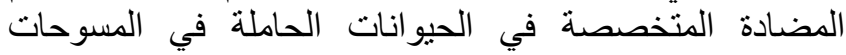
الوبائية (•(1). وهنالك طرق الكثر دقة في تشخيص الآنسابة

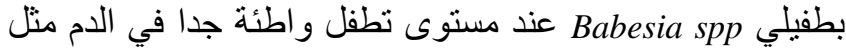

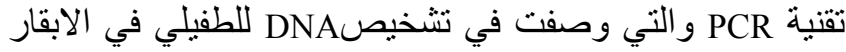

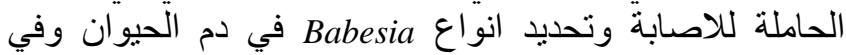

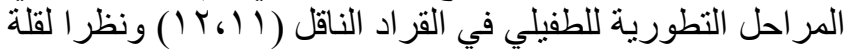

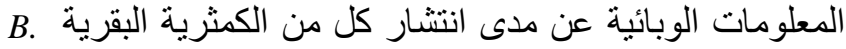

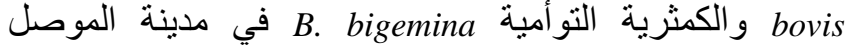
ولغرض دراسة الصفات الثكلية و القياسية وتحديد نسبة التطفل

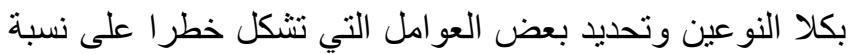
الإصابة مثل العمر و الجنس اجريت هذه العولي الدر اسة.

المواد وطر ائق العمل

جمع نماذج الام

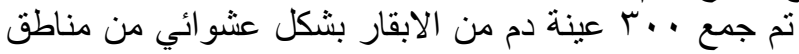

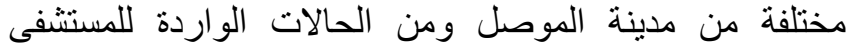




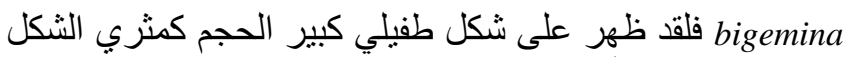

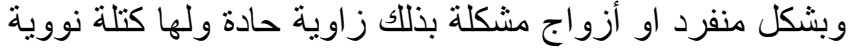

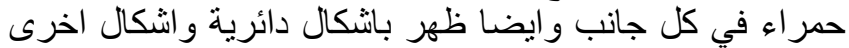

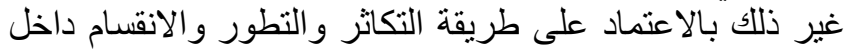

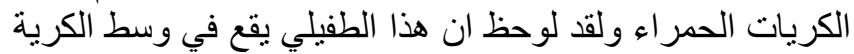

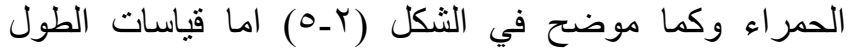

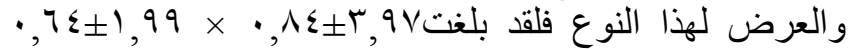

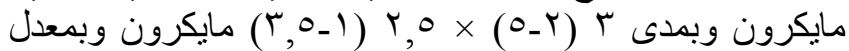

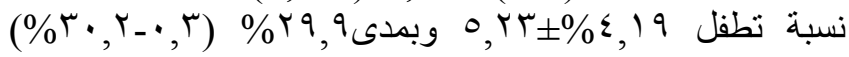

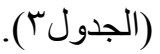

جدول 1: يبين أعداد ونسب الإصابة الكلية بكل من طفيلي و والنوعين Babesia spp و و B. bovis bovis المفحوصة

\begin{tabular}{|c|c|}
\hline r... & عدد الابقار المفحوصة \\
\hline ITV & Babesia spp عدد الابقار المصسابة ب \\
\hline س & Babesia spp نسبة الاصنابة الكلية بطفيلي \\
\hline 94 & B. bovis عدد العينات المصسابة ب \\
\hline$\%$ & نسبة الاصـابة \\
\hline r & B. bigemina عدد العينات المصسابة ب \\
\hline M & نسبة الاصسابة \\
\hline
\end{tabular}

جدول r : انماط الاصـابة بانواع Babesia spp

\begin{tabular}{|c|c|c|}
\hline الاصنبة \% & المصداب & نمط الاصسابة \\
\hline$\sum Y, 01$ & $0 \leqslant$ & Babesia bovis الاصـابة المفردة ب \\
\hline$r V, 00$ & ro & الاصـابة المفردة بـBabesia bigemina \\
\hline$r q, 9 r$ & ऍᄉ & الاصسابة المزدوجة بالنو عين \\
\hline 99,91 & ITV & المجموع \\
\hline
\end{tabular}

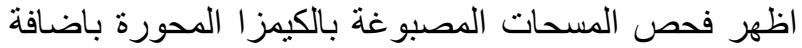

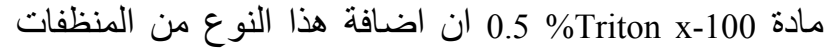
Detergents

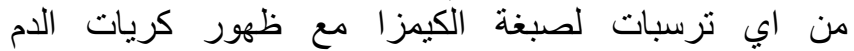

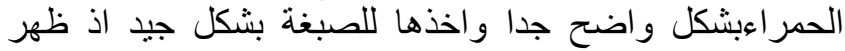

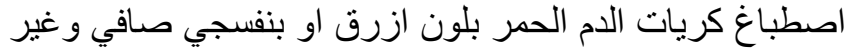
داكن او غامق مع ظهور طفيليات

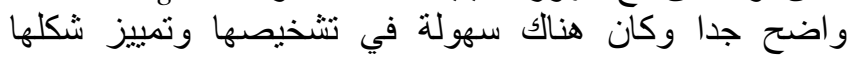

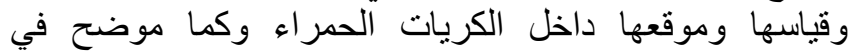

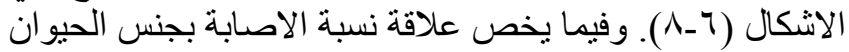

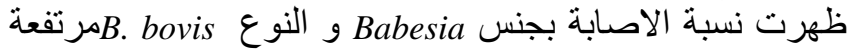

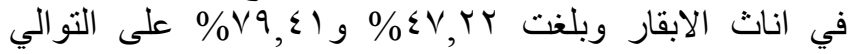

Ocular الطول و العرض لكل نوع باستخدام المقياس العيني micrometer

. (176, 10, r)

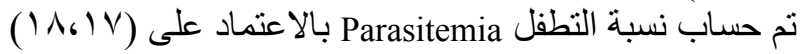

وفقا للمعادلة الاتية :

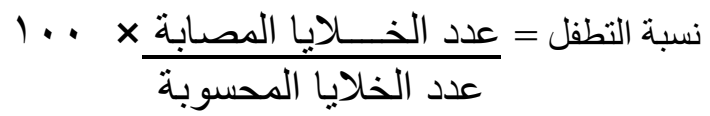

تحديد هل ان الحيوان مصاب ام غير مصاب بالطفيلي ينت

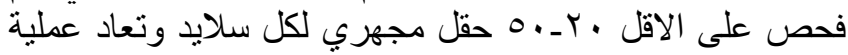

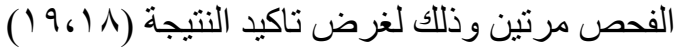

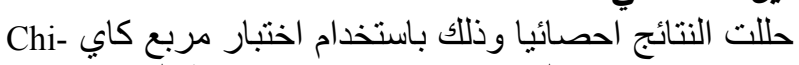

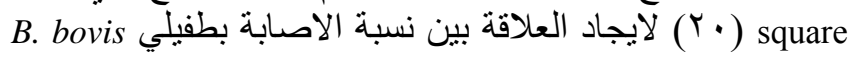

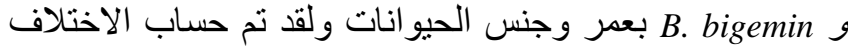

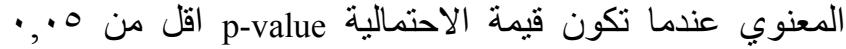

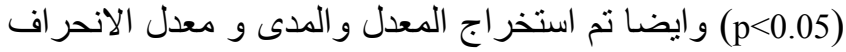

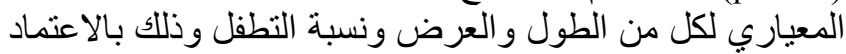

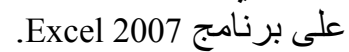

النتائج

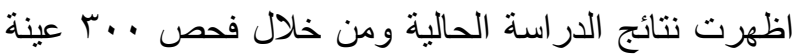

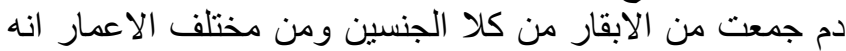

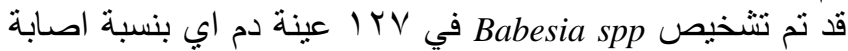

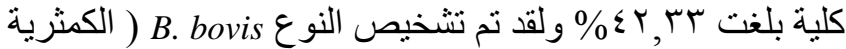

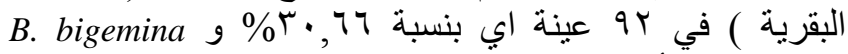

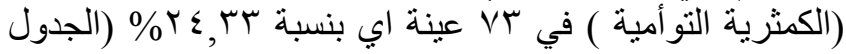

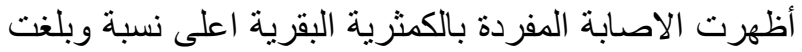

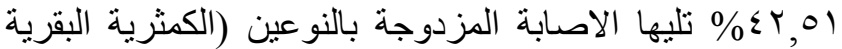

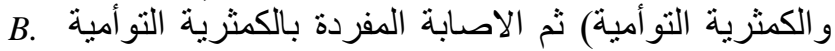
اذ بلغت النسب rigemina

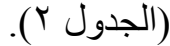

ظهر طفيلي Bovis في مسحات الدم الخفيفة على شكل طفيلي كمثري Pyriform صغير الحجم و غالبا ما يكون بشكل

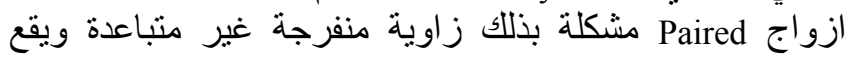

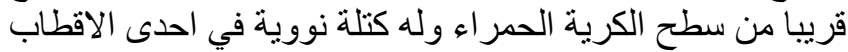

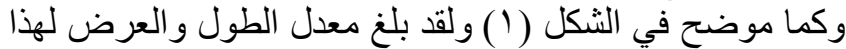

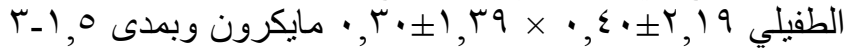

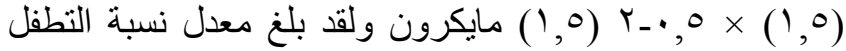

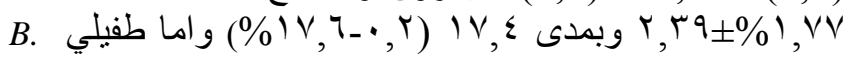




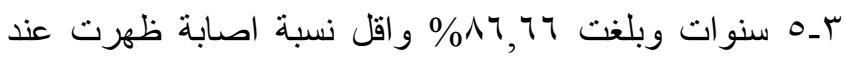

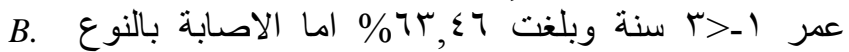
bigemina

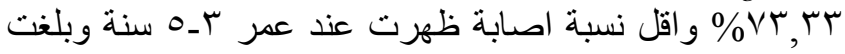

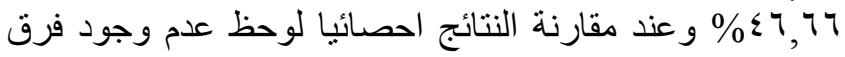

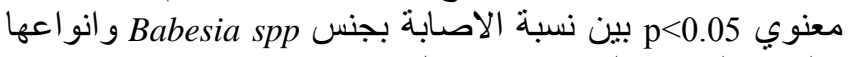

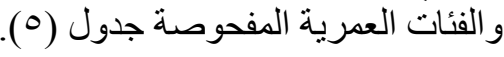

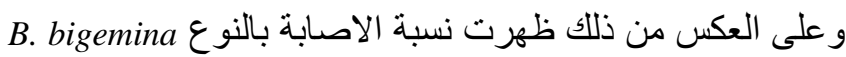

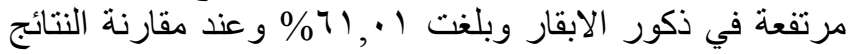

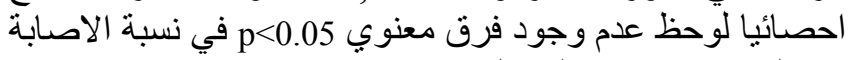

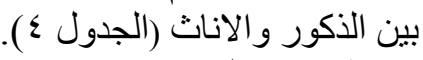

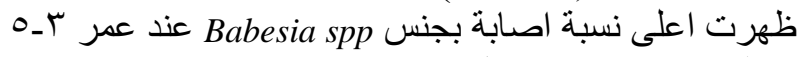

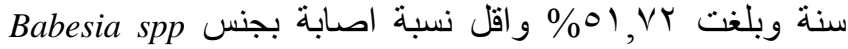

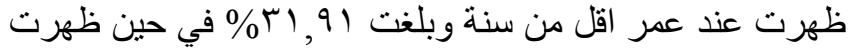

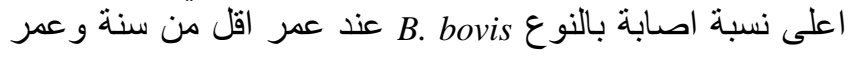

جدول r: يوضح المواصفات الثكلية والقياسية ونسبة التطفل لطفيلي Babesia bovis و Babesia bigemina المشخصة في عينات دم الابقار

\begin{tabular}{|c|c|c|c|c|c|c|c|}
\hline \multicolumn{2}{|c|}{ نسبة التطفل } & \multicolumn{2}{|c|}{ العرض (رm) } & \multicolumn{2}{|c|}{ الطول (رm) } & \multirow{2}{*}{ المو اصفات الثكلية } & \multirow{2}{*}{ الطفيلي } \\
\hline المدى & المعدل & المدى & المعدل & المدى & المعدل & & \\
\hline & & $\begin{array}{l}r_{-} \cdot, 0 \\
1,0=\end{array}$ & $\begin{array}{c} \pm 1, \mu q \\
\cdot r\end{array}$ & $\begin{array}{l}r-1,0 \\
1,0=\end{array}$ & $\begin{array}{c} \pm r, 19 \\
\cdot, \xi .\end{array}$ & 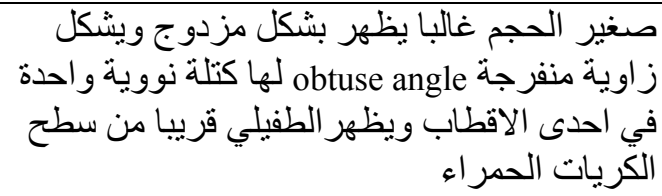 & $\begin{array}{c}\text { Babesia } \\
\text { bovis }\end{array}$ \\
\hline $\begin{array}{c}r \cdot r_{-} \cdot \vec{r}, r \\
r q, q=\end{array}$ & $\begin{array}{c} \pm \varepsilon, 19 \\
0, Y T\end{array}$ & $\begin{array}{l}r, 0_{-} 1 \\
r, 0=\end{array}$ & $\begin{array}{l} \pm 1,99 \\
\cdot, 7 \leqslant\end{array}$ & $\begin{array}{l}0_{-} r \\
r=\end{array}$ & $\begin{array}{l} \pm r, q \vee \\
\cdot, \wedge \varepsilon\end{array}$ & 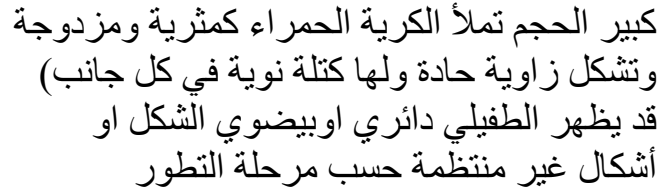 & $\begin{array}{c}\text { Babesia } \\
\text { bigemina }\end{array}$ \\
\hline
\end{tabular}

جدول ع : يبين علاقة نسبة الاصابة بجنسBabesia و النو عين Babesia bovis و Babesia bigemina بجنس الحيوان

\begin{tabular}{|c|c|c|c|c|}
\hline عدد العينات المصابة & عدد العينات المصابة & عدد العينات المصابة بجنس & عدد العينات & الجنس \\
\hline $\begin{array}{l}\text { ونسبة الاصـابة } \\
\text { ونسبة bigemina }\end{array}$ & $\begin{array}{c}\text { B. bovis } \\
\text { ونسبة الاصـابة }\end{array}$ & $\begin{array}{c}\text { Babesia spp } \\
\text { ونسبة الاصـابة }\end{array}$ & المفحوصة & \\
\hline$C(0 \leqslant, \leqslant)) \Gamma V$ & $\mathrm{~B}(\vee q, \leqslant)) \circ \varepsilon$ & $A(\Sigma V, r Y) T \Lambda$ & $1 \leq \varepsilon$ & الاناث \\
\hline C & $\mathrm{B}(\tau \Sigma, \varepsilon \cdot) \Gamma \Lambda$ & $A(r V, \wedge r) \circ q$ & 107 & 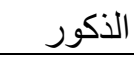 \\
\hline$(T \Sigma, r r) \vee r$ & $(r \cdot, 74) 9 r$ & $(\varepsilon r, r T) I T V$ & r... & المجموع \\
\hline
\end{tabular}

جدول ๑: يبين علاقة الاصابة بكل من Babesia bovis مبعمر الابقار Babesia bigemina

\begin{tabular}{|c|c|c|c|c|}
\hline عدد العينات المصسابة & عدد العينات المصسابة & عدد العينات المصابة بجنس & عدد العينات & العمر \\
\hline 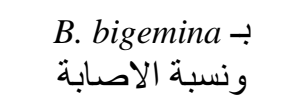 & $\begin{array}{c}\text { B. bovis بـ ونسبة الاصنة } \\
\text { ونسبة }\end{array}$ & $\begin{array}{c}\text { Babesia spp } \\
\text { ونسبة الاصـابة }\end{array}$ & المفحوصة & \\
\hline C (VT,rr) & $B(\wedge \neg, \neg 7) \backslash \mu$ & $A(r), q 1) 10$ & $\varepsilon V$ & اقل من سنة \\
\hline$C(00, V 7) r q$ & מז & $A(\varepsilon \cdot, 9 \varepsilon)$ or & IrV & $r \geq 1$ \\
\hline$C(\leq 7,77) \vee$ & $\mathrm{B}(\wedge 7,77) ו \pi$ & $A(01, V Y) 10$ & rq & $\overline{0-4}$ \\
\hline$C(O V, V V) r T$ & מ & $A(\leq \tau, r q) \leq 0$ & $9 \vee$ & هنو ات فاكبر \\
\hline$(O V, \leqslant \Lambda) \vee r$ & $(V Y, \Sigma \varepsilon) 9 Y$ & $(\varepsilon r, r T) I T V$ & r.. & المجموع \\
\hline
\end{tabular}




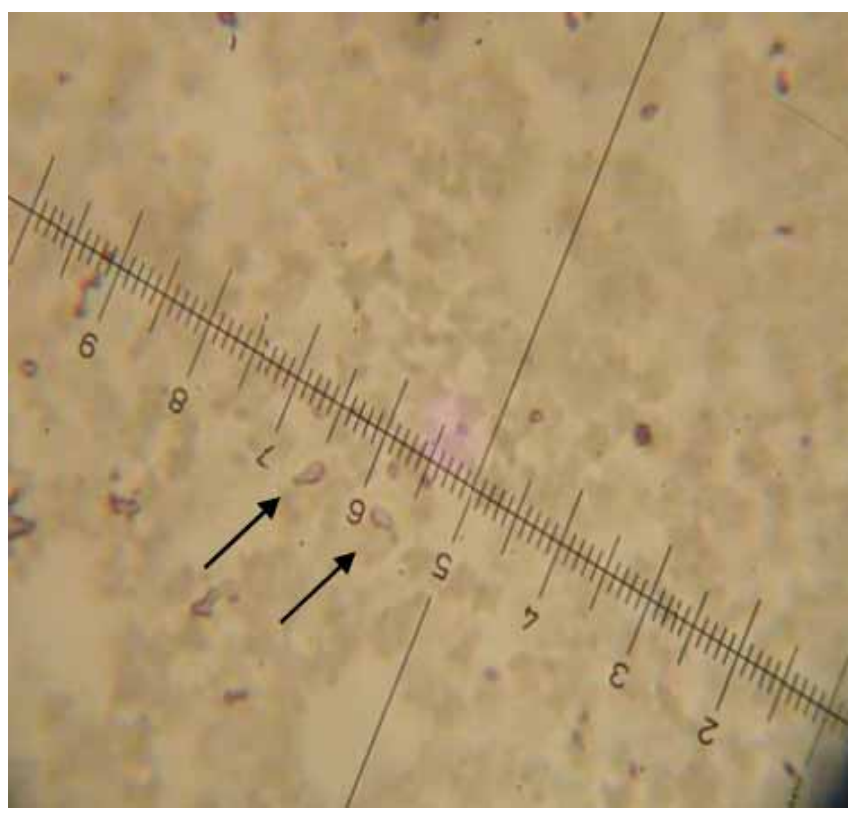

B. bigemina شكل r: يوضح الشكل الكمثري والمنفرد لطفيلي (1) $(1)$

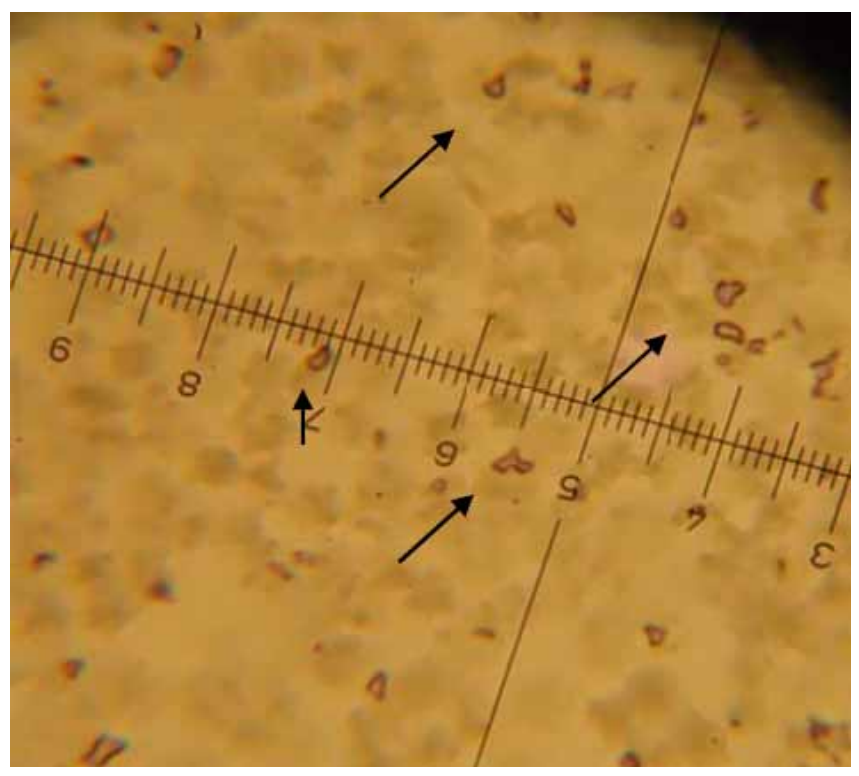

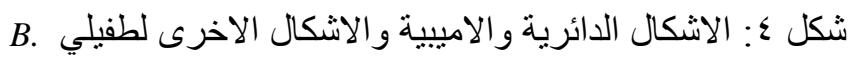
bigemina

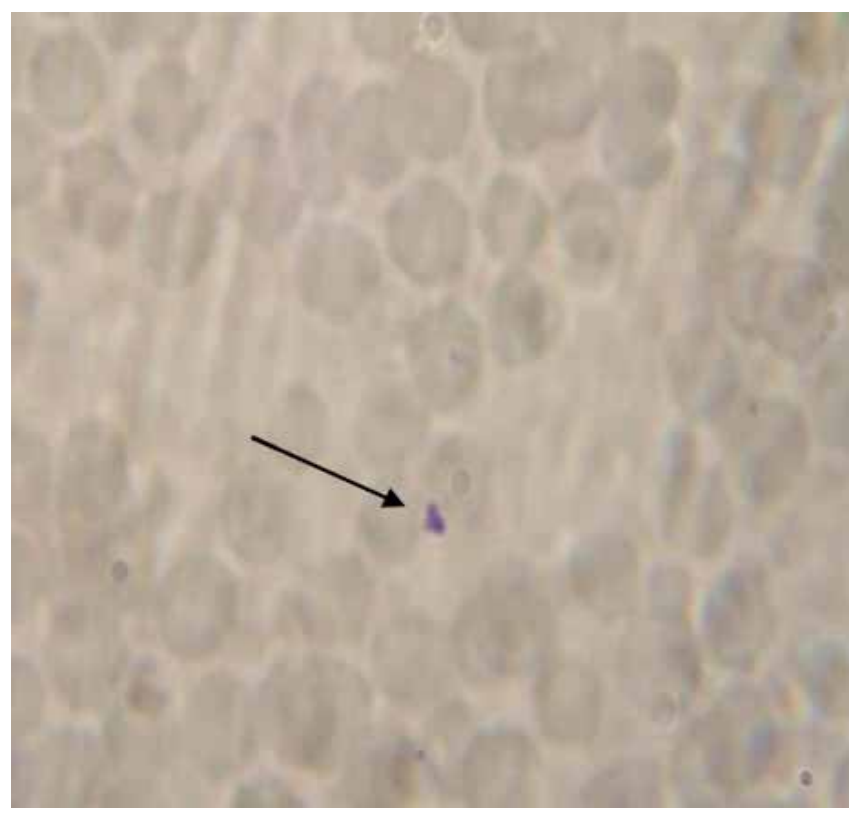

شكل 1: طفيلي B. bovis ( . (1).

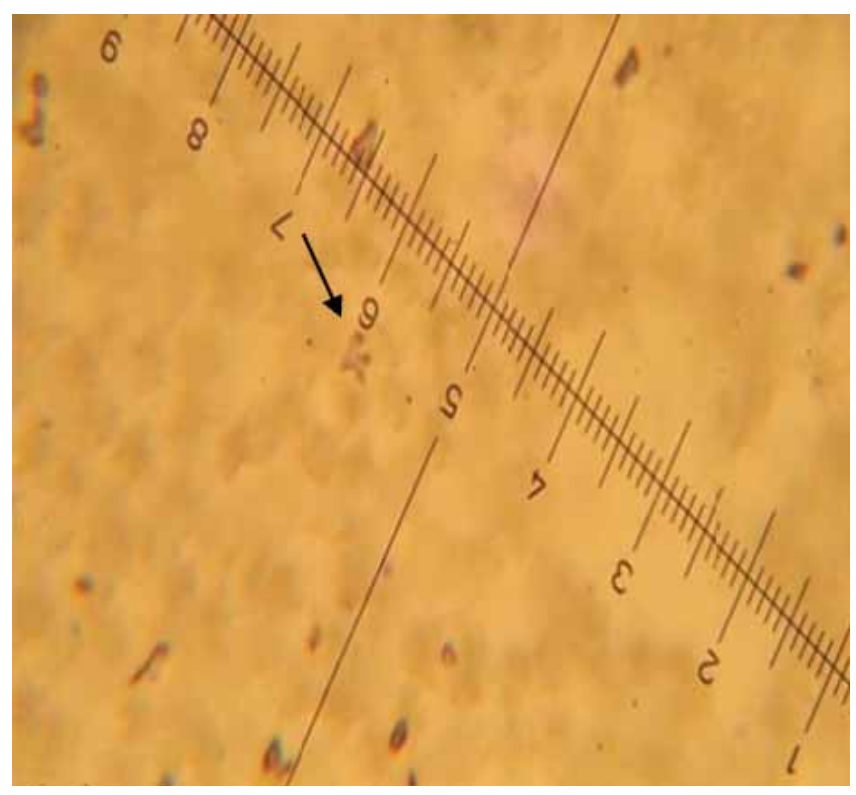

شكل ؟: طريقة تكاثر طفيلي B. bigemina في كريات الدم

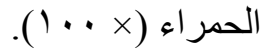




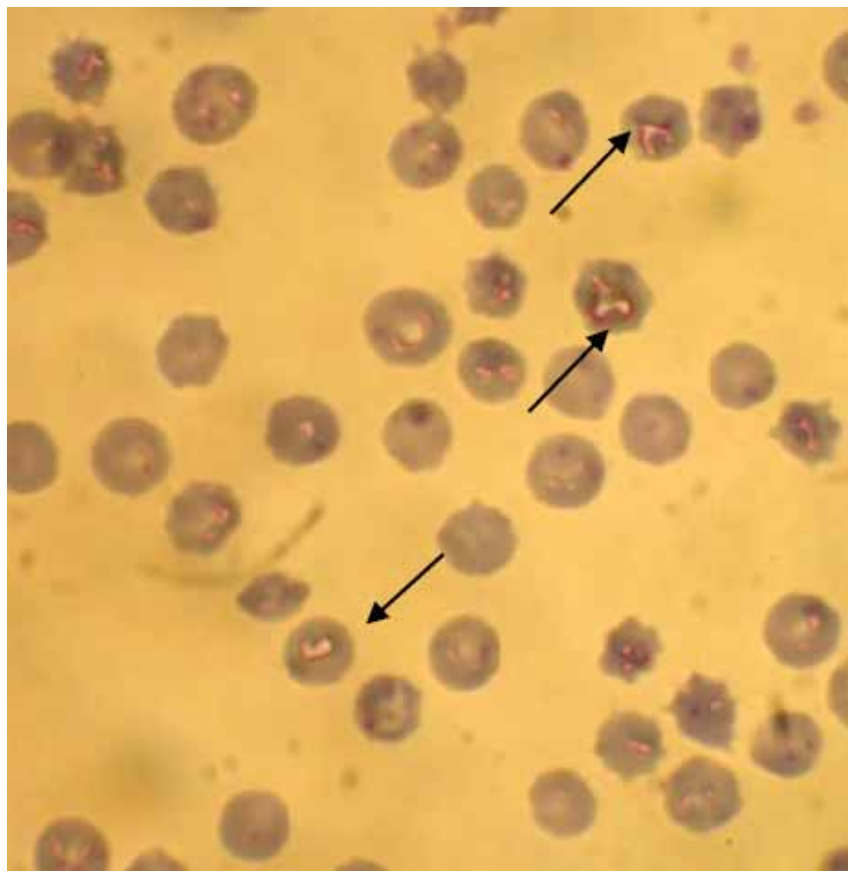

شكل \: يوضح طريقة تكاثر لطفيلي B. bigemina داخل كريات

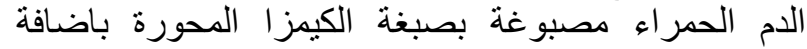
.( ( . × $0.5 \%$ TritonX-100

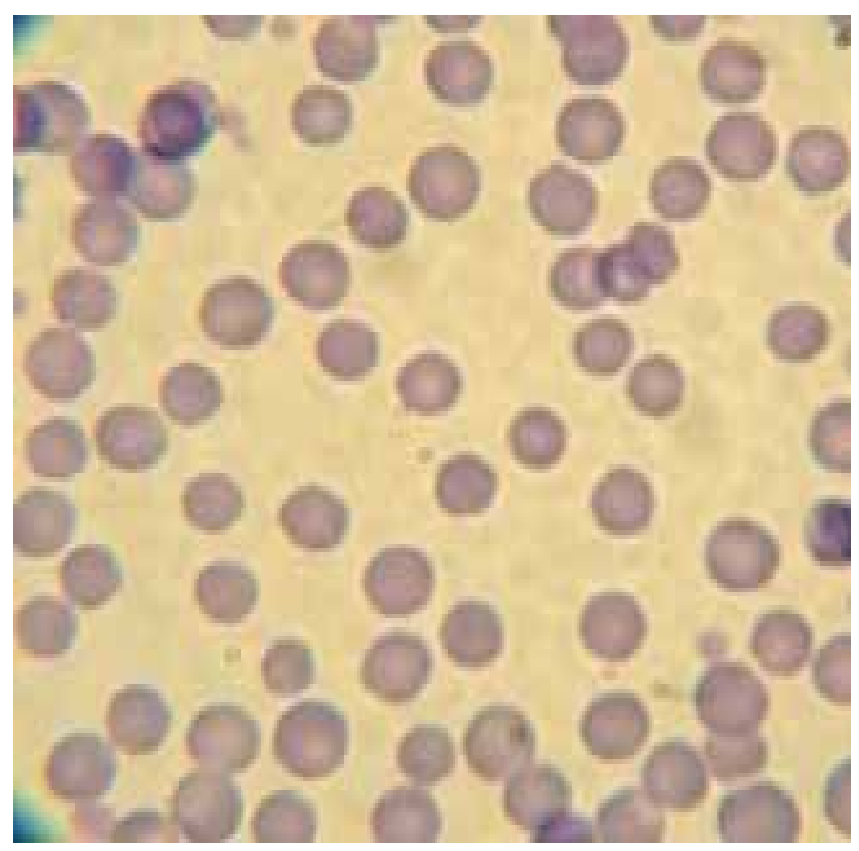

شكل ^: يوضح شكل وصبغ كريات الدم الحمر اء للابقار بصبغة الكيمز المحورة باضافة

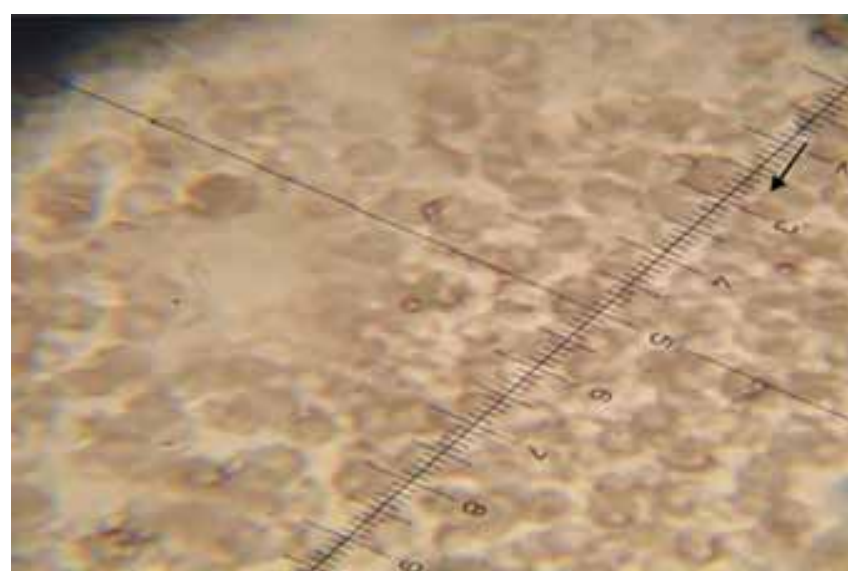

شكل ๑: يوضح الشكل المزدوج لطفيلي B. bigemina (× ( )..

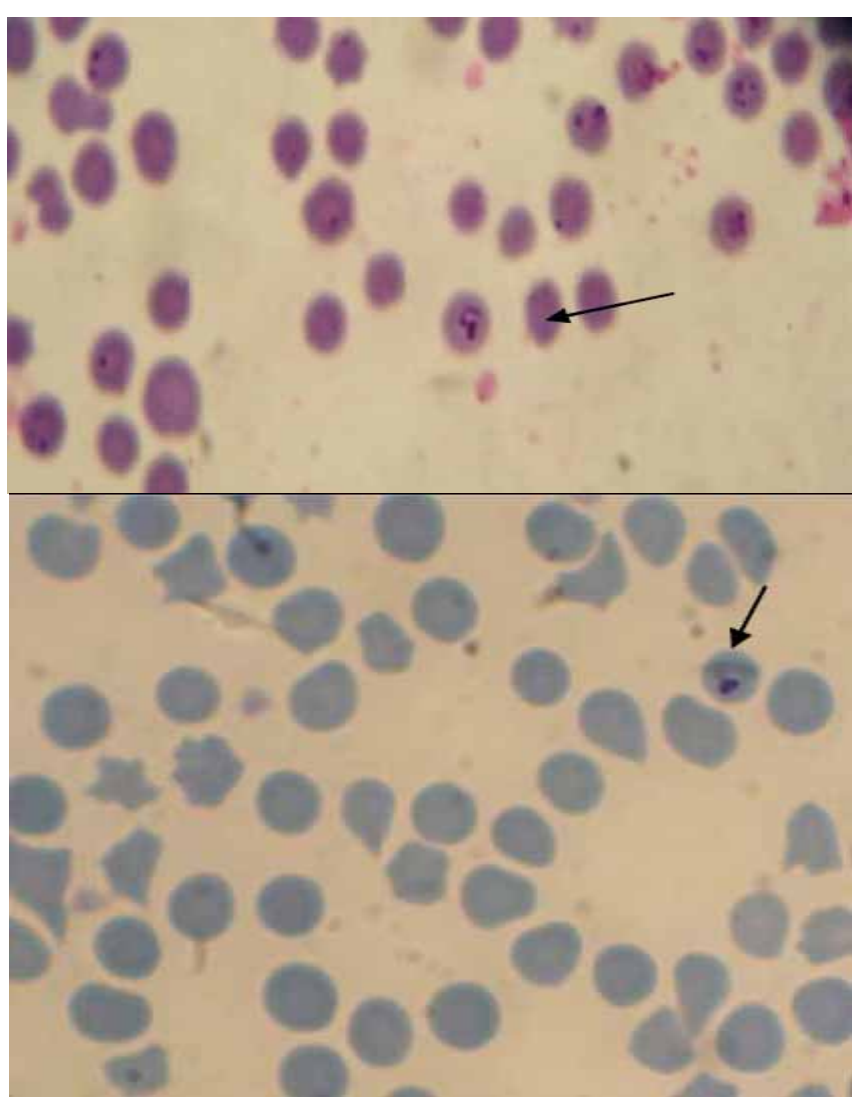

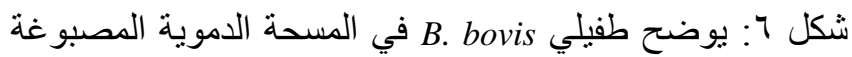
بصبغة الكيمز ا المحورة بإضافة 


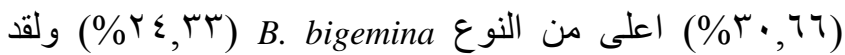

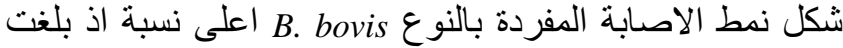

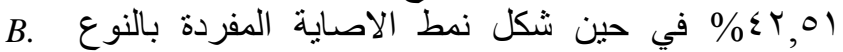

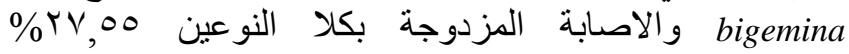

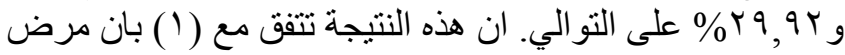

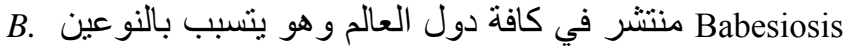
B. bigemina gbovis وان لهما انتشارواسع بسبب الانس النتشار

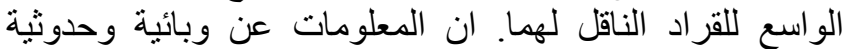

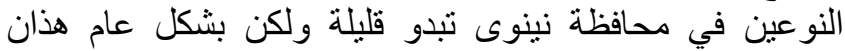

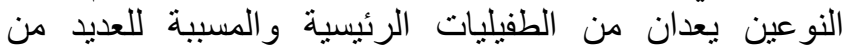

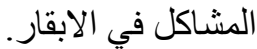

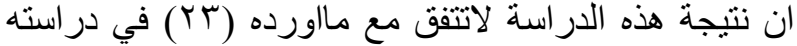

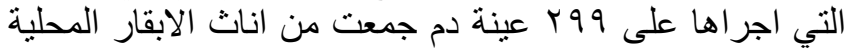

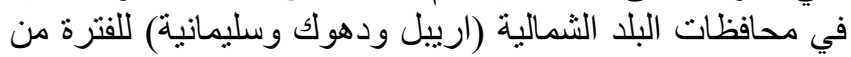

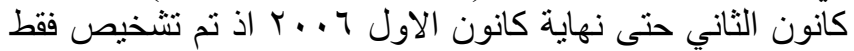

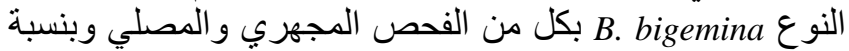

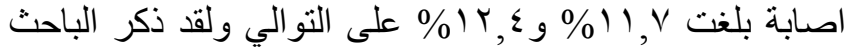

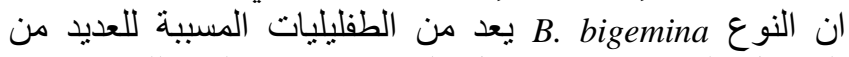

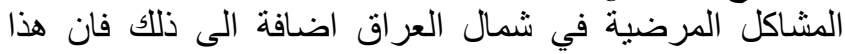

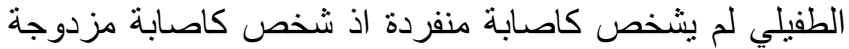

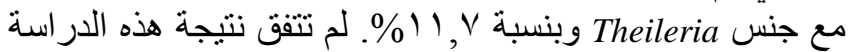

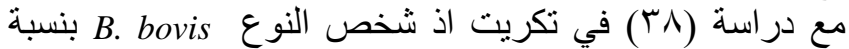

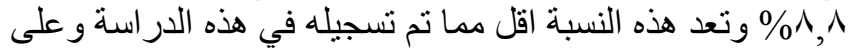

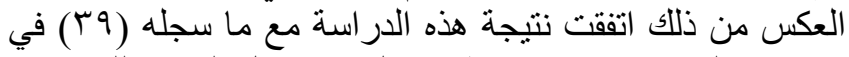

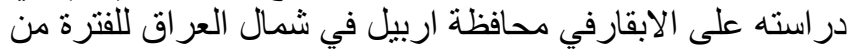

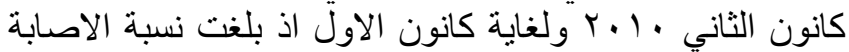

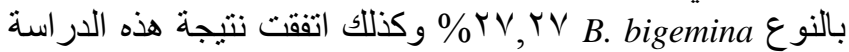

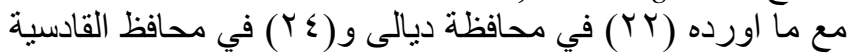

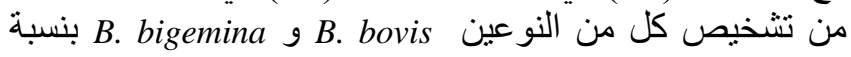

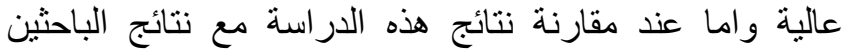

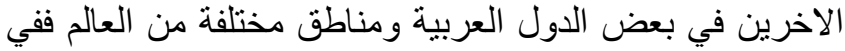

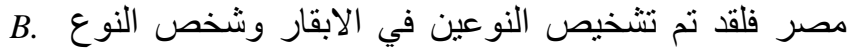

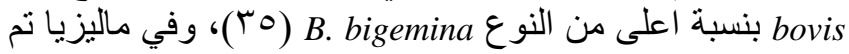

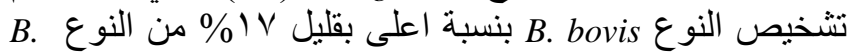
bigemina

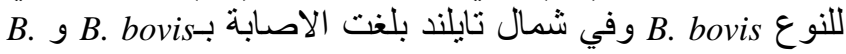

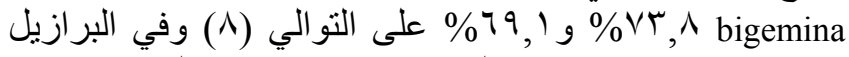

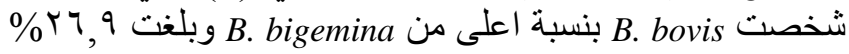

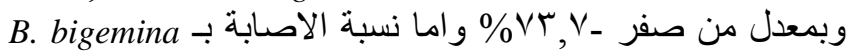

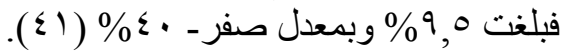

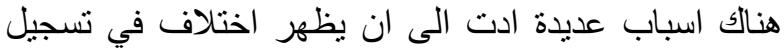

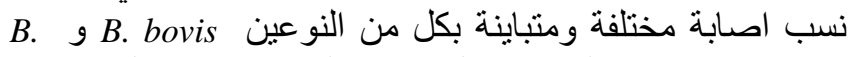
bigemina

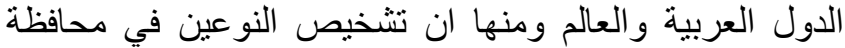
نينوى يرجع الى الانتشار الواسع للقراد وهذا يتفق مع ماذئ فئره

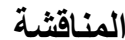

اوضحت نتائج الدراسة الحالية ان نسبة الاصابة الكلية

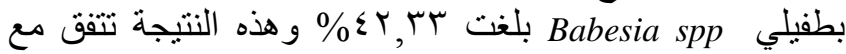

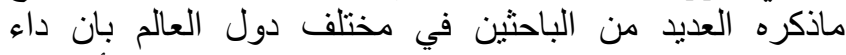

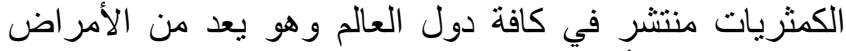

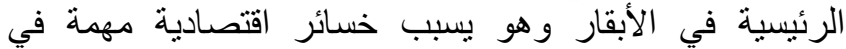

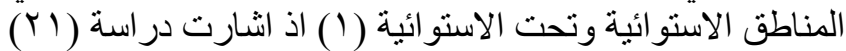

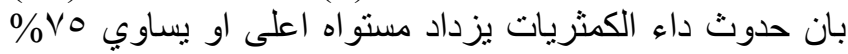

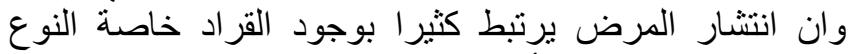
Boophilus microplus

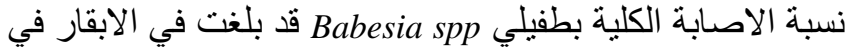

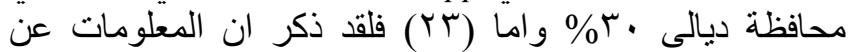

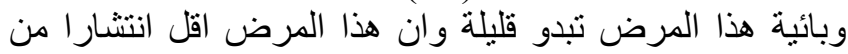

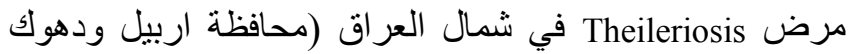

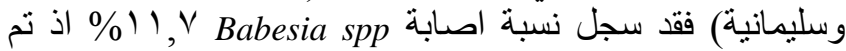

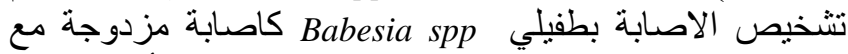

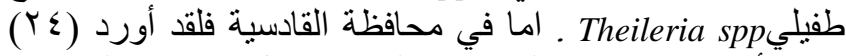

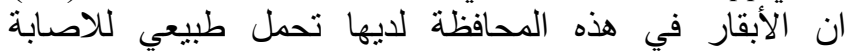

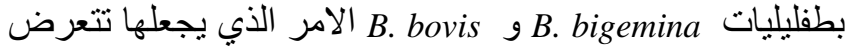

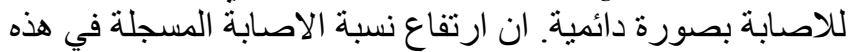

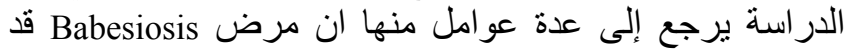

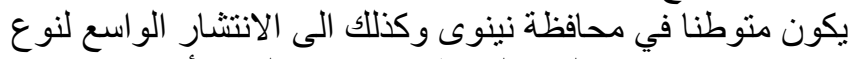

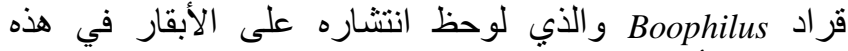

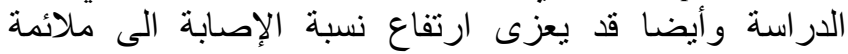

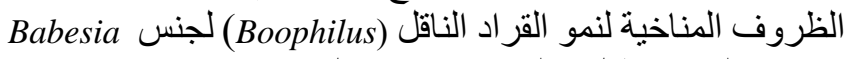

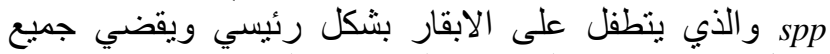

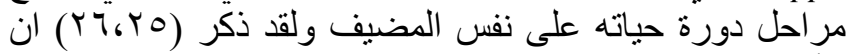
الأمر اض المنسبية بالطفيليات الدموية لها علاقة علفة معنوية مع وجود

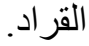

اتفقت نتيجة هذه الدراسة مع ماسجله عدد من الباحثين في

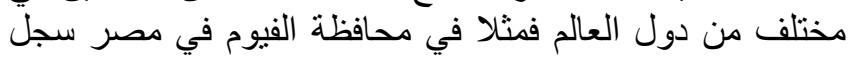

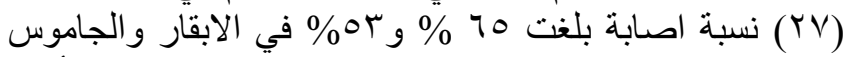

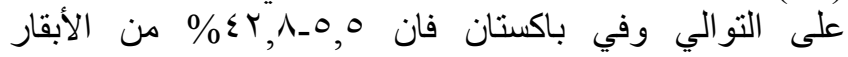

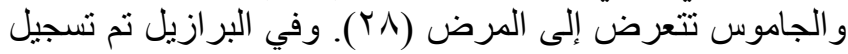

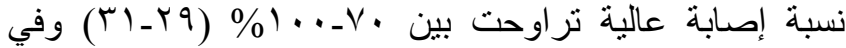

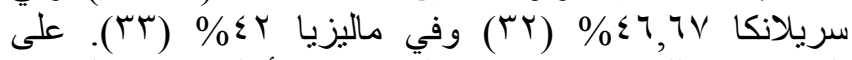

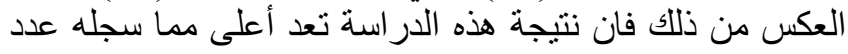

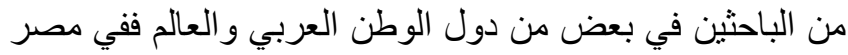

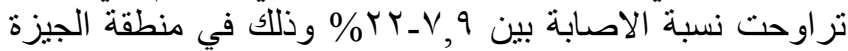

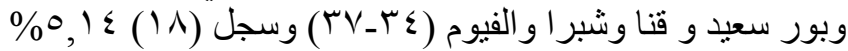

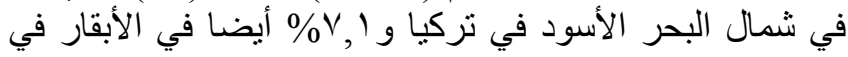

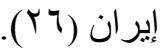

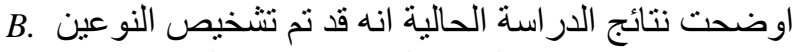

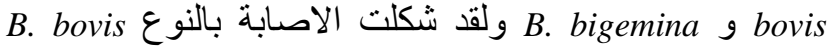


للاصابة خاصة في المناطق التي يكون فيها المرض منوطنا

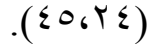

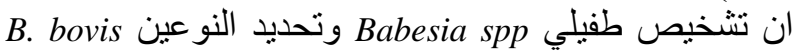

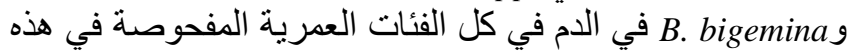

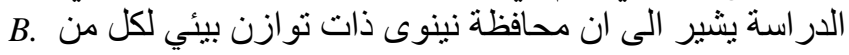
د. bovis gbigemina

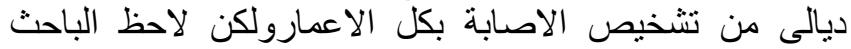

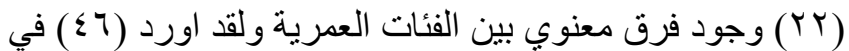
محافظة النجف بان طفيليات الدم تحدث في جميع الاعمار وان

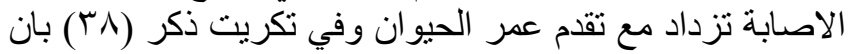

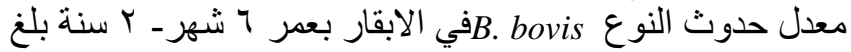
\%^, ,

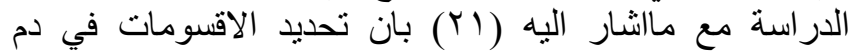

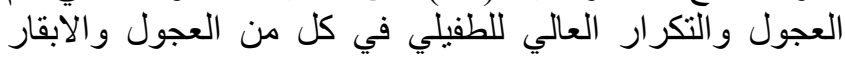

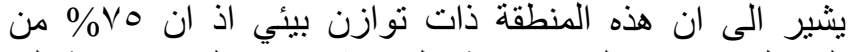

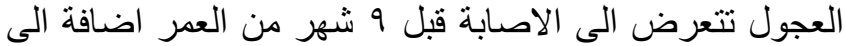

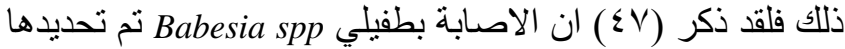

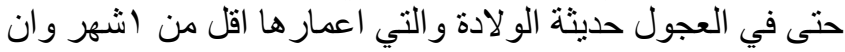

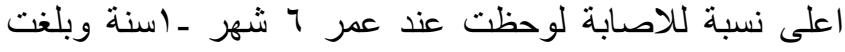

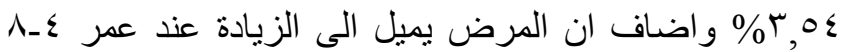

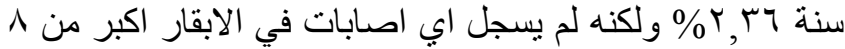

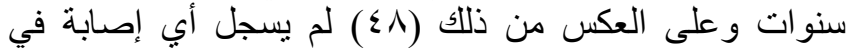

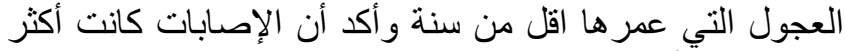

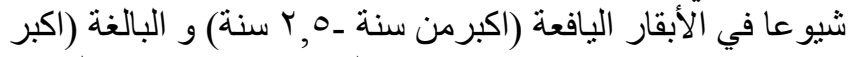

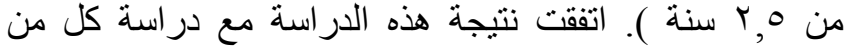

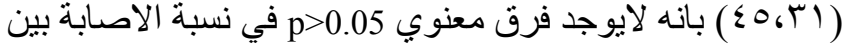

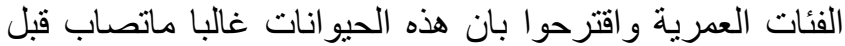

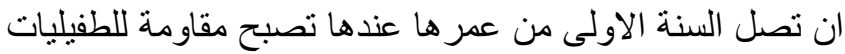

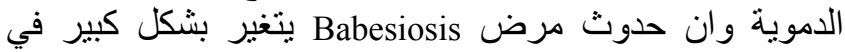

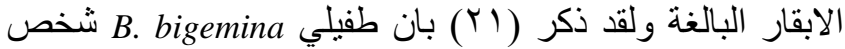

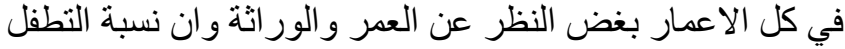

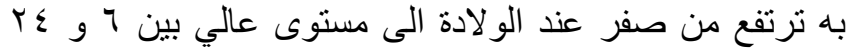

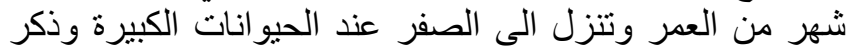

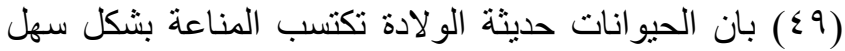
Passively immunity

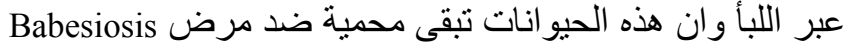

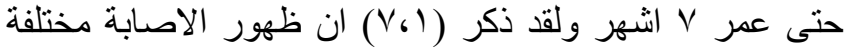

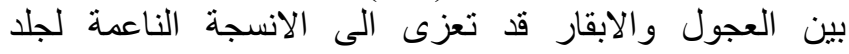

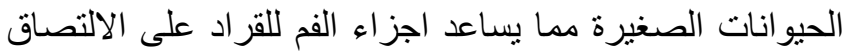
و التغذية بسهولة ونقل المرض.

المصادر

1. Bock R, Jackson L, Devos A, Jorgensen W. Babesiosis of Cattle. Parasitol. 2004;129:247-269.

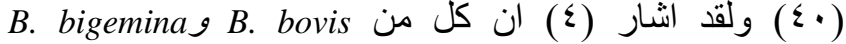
يتو اجدان في نفس المو اقع الجغرافية ولكن توجد اختلافات قليلة

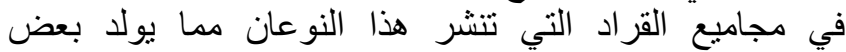

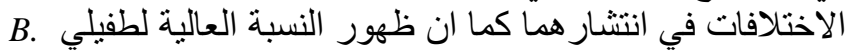

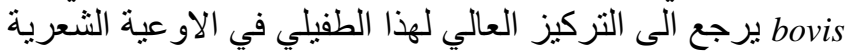

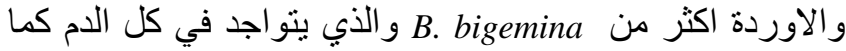

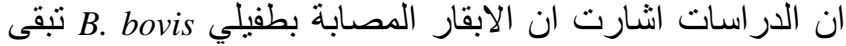

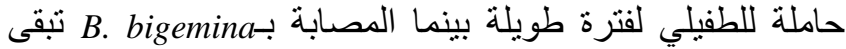

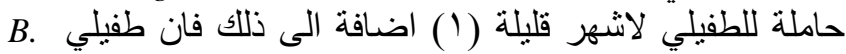

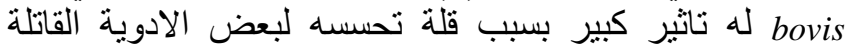

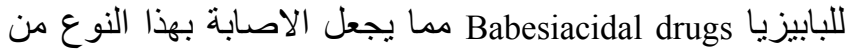

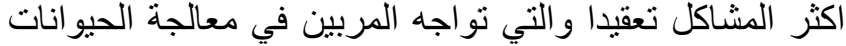

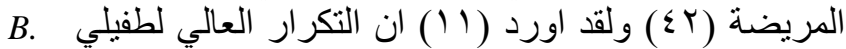
bovis و غياب السيطرة على المرض. تم تشخيص طفيلي B. bovis ماستخدام

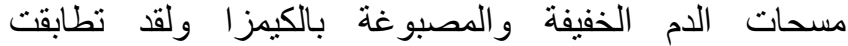

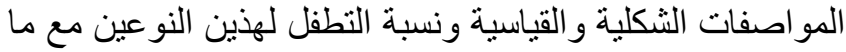

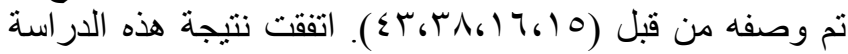

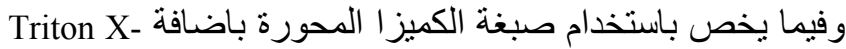

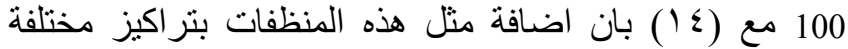

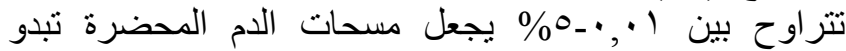
واضحة جدا وتعطي اصطباغا جيدا للطفيليات الدموية.

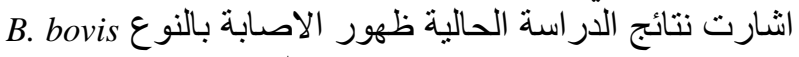

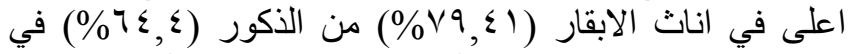

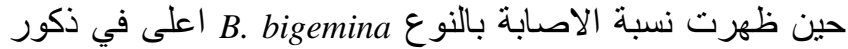

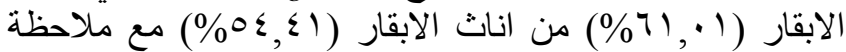

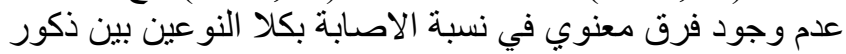

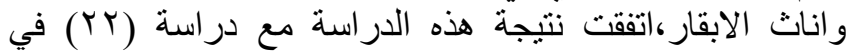
محافظة ديالى و(؟Y Y) في محافظة القادية. انثارت العديد من الدر اسات في مختلف دول العالم بان الاناث

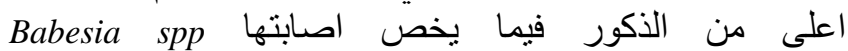

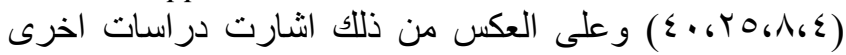

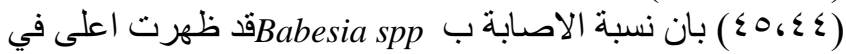

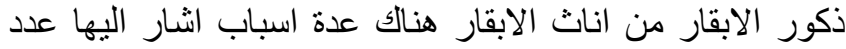

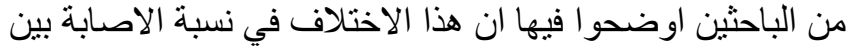

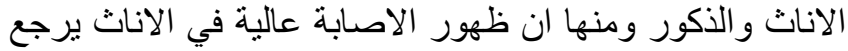

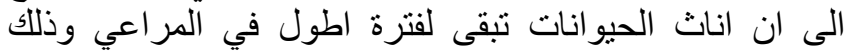

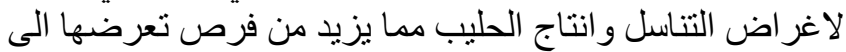

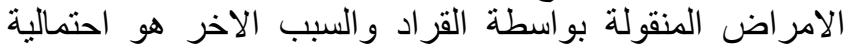

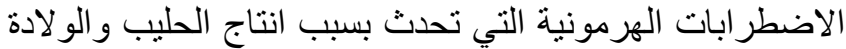

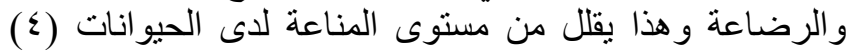

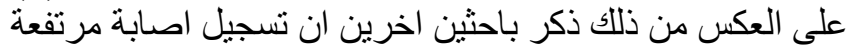

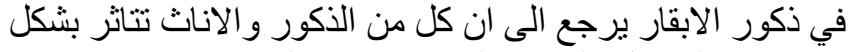
متساوي بالعو امل الممهدة للاصابة وان لهان لهما نفس الاستعدادية الانية 


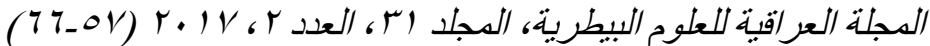

23. Omer LT, Kadir MA, Ahmed JS. Seroprevalence of piroplasmosis with tick distribution in northern Iraq. Iraqi J Vet Sci. 2012;26 (Supplement III): 105-108.

24. Sabber KH,Aaiz NN. Molecular detection of Babesia bovis in cattle in AL-Qadisiyah Province. Iraqi J Vet Sci. 2016;40(2):155-158.

25. Lemma F,Girma A,Demam. D.Prevalence of babesiosis in and around Jimma town western in Ethiopia. Adv Biolo Res. 2016;10(1):37-42.

26. Khamesipour F,Doosti A,Koohi A, Chehelgerdi M. Determination of the presence of Babesia DNA in blood samples of cattle, camel and sheep in Iran by PCR. Arch Biol Sci Belgrade. 2015;67(1):83-90.

27. El-Bahy NMA.Some studies on ticks and tick borne disease among ruminants in Fayom governorate.M.V.Sc.thesis (parasitology ) faculty of veterinary medicine ,Cairo University, Cairo, Egypt Google Scholar.1986.

28. Niazi N, Khan MS, Avais M, Khan JA, Pervez K,Ijaz M. A study on Babesiosis in calves at livestock experimental station Qadirabad and adjacent areas Sahiwal, Pakistan. Pak J Agri Sci. 2008;45:13-16.

29. Oliveira-Sequeira TCG, Oliveira MCS, Aranjo JP,Amarante AFT. PCR-based detection of Babesia bovis and Babesia bigemina in their natural host Boophilus microplus and cattle. Internat J Parasitol. 2005; 35(105-111).

30. Bell SL,Koney EB,Dogbey O, Walker AR. Incidence and prevalence of tick -borne haemoparasires in domestic ruminants In Ghana. Vet Parasitol.2004; 124:25-42

31. Souza FA,Braga JFV,Pires LV,Carvalho CLS, Costa AE, Riberio MFB, Santoes RL, Silva MMS. Babesiosis and anaplasmosis in dairy cattle in Northeastern in Brazil. PesqVet Bras.2013;33(9):1057-1061.

32. Kirupananthan R, Kamarall CJ,Galhena GH, Perera KLN ,Magamage MIPS.Address the public health and food security concerns of babesiosis through molecular detection of Babesia bovis in suspected carrier cattle of selected localities in Sri Lanka. Procedia Food Sci.2016;6:213-219.

33. Rahman WA, Lye YP,Chandrawathani P.The seroprevalence of bovine babesiosis in Malaysian.TROP Biomed. 2010;27:301-307.

34. Ibrahim AK, El Behairy AM, Mahran KA, Awad WS. Clinical and Laboratory diagnosis of piroplasmide in naturally infected cattle in Egypt. J Egypt Vet Med Assoc. 2009;69(2):105-203.

35. El- Fayomy AO, Ghoneim AM, Abu-Samak OA, Khidr AA. Contribution of Babesia to the Illness of cows in Port Said Governorates ,Egypt. Global Veterinaria. 2013;11(1):118-122

36. NayelM, El-Dakhly KM, Mohamed K, Aboulaila M, Elsify A Hassan H, Ibrahim E, Salama A ,Yanai T. The use of different diagnostic tools for Babesia and Theileria parasites in cattle in Menofia. Egypt Parasit Res. 2012;111(3):1019-1024

37. Hossary AAT. Comparism between conventional and molecular methods for diagnosis of bovine babesiosis (Babesia bovis infection ) in ticks infested cattle in upper Egypt. J Parasite Dis. 2016;DOI 10.1007/s12639-016-0785-2

38. Ibrahim O ,Taha Z, Jassim S. Prevalence of Babesia bovis in cattle in Tikreet city and its surroundings with hematological study.Tikreet $\mathbf{J}$ Pure Sci. 2012;17(2):32-34

39. Ameen KAH,Abdullah BA,Abdul-Razaq.Seroprevalence of Babesia bigemina and Anaplasma marginale in domestic animals in Erbil,Iraq. Iraqi Journal of Veterinary Science.2012; 26 ,Supplement III 109-114

40. Khan A, Rehman AU, Hisham M, Khan A, Rahman HU, Khan MI, MF Q and Ameen MA. Burden of babesiosis among domestic cattle of southern Khyber Pakhtunkhwa. Pakistan J Entomol Zool Stud. 2016;4(5):305-307.

41. Costa VMJM, Ribeiro MFB, Duarte AL. Seroprevalence and risk factors for cattle anaplasmosis, babesiosis and trypanosomiasis in a Brazilian semiarid region. Rev Bras Parasitol. Vet J botic bat, 2013; 22(2): 207-213.

42. Uilenberg G. Babesia - A historical overview. Vet Parasitol. 2006;138:3-10.

43. Hussain S,Ashraf ,K,ANWAR N,Jamal MA, Naeem H, Ahmad N ,Rahman AU. Diagnosis of Babesia infection in Iindigenous and
2. World Organization for animal Health (OIE). Manual of diagnostic tests and vaccines (online), Paris, Bovine babesiosis: http://222.oie.leng.inurmes/mannal. 2008/pdf12.04.0.

3. Chaudhry ZI, suleman M, Younus M, Aslim A. Molecular detection of Babesia bigemina and Babesia bovis in Crossbred carrier cattle through PCR. Pakistan J Zool. 2010; 42(2):201-204.

4. Hamsho A, Tesfamarym G,Megersa G,Megersa M.A cross - Sectional of Bovine babesiosis in Teltele district ,Borena ,zone ,Southern Ethiopia. Vet Sci Technol. 2015;6(3)http://dx.doi.org/10.4172-21577579.1000230

5. Friedhoff KT.Transmission of Babesia ,In Babesiosis of domestic animals and man (ed Ristic. M.), PP. 23-52. Boca Raton. Florida, CRC Press. Inc. 1988

6. Bhata SA, Singha NK, Singh HA,Ratha SS. Molecular prevalence of Babesia bigemina in Rhipicephalus microplus ticks infesting crossbred cattle of Punjab, India. Parasite Epidemiol Cont. 2017;2:85-90.

7. Zulfiqar S, Shahnawaz S, Ali M, Bhuta AM, Iqbal S, Hyat S, Qadir S, Latif M, Kiran N, Sad A, Ali M, Iqbal F.Detection of Babesia bovis in blood samples and its effect on the hematological and serum biochemical profile in large ruminants from Southern Punjab. Asian Pacific J Trop Biomed. 2012;104-108.

8. Terkawi MA, Huyen NX, Shinuo C, Impankaew, Maklon K, Aboulaila M, Uno A, Goo YK, Yakoyama N, JiHapalapong S, Xuan $\mathrm{X}$, Igarashi I. Molecular and Serological prevalence of Babesia bovis and Babesia bigemina in water buffaloes in the northeast region of Thailand. Vet parasitol. $2001 ; 178: 201-207$.

9. Demessie Y and Derso S. Ticks borne hemoparasitic diseases of ruminants :A Review. Adv Biolo Res.2015;9(4):210-224.

10. Silva J, Andre MR, Fonseca AH, Lopes CIA, Lima DHS, Andrade SJT, Oliveria CMC, Barbosa JD. Molecular and serological prevalence of Babesia bovis and Babesia bigemina in water buffaloes in the north region of Brazil. 2013: http://dx.doi.org/10.1016/j.vetpar.2013.05.02022

11. Martins TM, Neves L, Pedro OC, Facetine JM, Dorosario EV ,Domingos A. Molecular detection of Babesia spp and other haemoparasitic infections of cattle in Maputo Province, Mozambique. Parasitol. 2010;1-8.

12. Ramos CM, Cooper SM, Holman PD. Molecular and serologic evidence for Babesia bovis - like parasites in white-tailed deer Codocoileus virginianus in south Texas. Vet Parasitol. 2010;172:214220.

13. Hendrix CM. Diagnostic Veterinary Parasitology. 2nd Ed., Mosby Inc., USA, 1999; 164-227.

14. Melvin MD and Brooke.Triton X -100 in Giemsa staining of blood parasite.Biote Histochem.1995;30(6):269-275.

15. Soulsby EJL. Helminths, arthropds and protozoa of domesticated animals. 7th ed., Philadelphia, Bailliere Tindall, London, 1986;pp:707-716.

16. Urquhart GM, Armour J,Duncan JL, Dunn AM, and Jennings FW, Veterinary Parasitology. 2nd ed., Black Well Science 2003. 242-245.

17. Jain NC. Schalm's Veterinary hematology 4th. Ed. Lea and Febiger Philadelphia 1986; pp:610-612.

18. Altay K, Aydin MF, Dumanli N, Aktas M. Molecular detection of Theileria and Babesia infection in cattle. Vet Parasitol. 2008;158:295301.

19. Mahmmod YS. Molecular detection of natural Babesia bovis infection from clinically infected and apparently healthy water Buffaloes (Bubalus bubalis) and crossbred cattle. J Buffalo Sci. 2012;1: 55-60.

20. Petrie WP.Statistics for veterinary and animal science.BlackwellScience.London.2003;pp:101-113

21. Mahoney DF and Ross DR. Epizootiological Factors in the control to bovine babesiosis. Aus Vet J. 1972;48:292-298.

22. Minnat TR, Zuhairi AH, Jalil WI.Sudy of clinical ,epidemiological and heamatological changes of heamoparasites infection in cattle of Diyala province -Iraq. Res J Pharmaceut Biolod Chem Sci..2016;7(4): 3094. 
47. Muraleedharan K.Babesia and babesiosis of Karanaaka state.India An overview. Vet Res Internat. 2015;3(4)81-88.

48. LorussoV,Wijnveld M, Majekoudnmi A ,Dongkum C,Fajinmi A,Dogo A,Thrusfielield M,Mugeny I A, Vaumourin E ,lgweh A.Jongejan F, Welburn S,Picozzi K. Tick -borne pathogens of zoonotic and veterinary importance in Nigerian cattle. Parasit Vect.2016;9:217.

49. Brown WC, Norimine J, Knowles DP, Goff WL. Immune control of Babesia bovis infection Vet Parasitol. 2006;138: 75-87. crossbred cattle with comparison between conventional and molecular diagnostic techniques. J Infe Molec Biol. 2017;5(1)1-6.

44. Onoja II, Malachy., Mshelia W, Okayeto SO, Danbirni S. Kwanashie G. Prevalence of Babesiosis in cattle and goats at Zaria abattoir. Nigeria J Vet Adv. 2013;3(7):211-214.

45. Wobo SOS , Uyigue J, Surakat OA, Adekunle NO, Magoaji HO. Babesiosis and other haemoparasitic disease in a cattle slaughtering abattoir in Abeokuta ,Nigeria. Internat J Trop Dis Health. 2016; 18(2)1-5.

46. Al-Amery A, Faraj AA,. Majeed AS. Detection of hemoprptozoa in camel in AL-Najf province, Iraq. IJABR.2017;7(2): 238-24. 